\title{
The minimal curvature of the universe in modified gravity and conformal anomaly resolution of the instabilities
}

\author{
Shin'ichi Nojiri ${ }^{\star 1}$ and Sergei D. Odintsov ${ }^{\mathrm{P} 2}$ \\ - Department of Applied Physics, National Defence Academy, \\ Hashirimizu Yokosuka 239-8686, JAPAN \\ $\checkmark$ Institut d'Estudis Espacials de Catalunya (IEEC), \\ Edifici Nexus, Gran Capità 2-4, 08034 Barcelona, SPAIN \\ \& Instituciò Catalana de Recerca i Estudis Avançats (ICREA), Barcelona, \\ SPAIN
}

\begin{abstract}
We discuss the modified gravity which may produce the current cosmic acceleration of the universe and eliminates the need for dark energy. It is shown that such models where the action quickly grows with the decrease of the curvature define the FRW universe with the minimal curvature. It is required the infinite time to reach the minimal curvature during the universe evolution. It is demonstrated that quantum effects of conformal fields may strongly suppress the instabilities discovered in modified gravity. We also briefly speculate on the modification of gravity combined with the presence of the cosmological constant dark energy.
\end{abstract}

PACS numbers: 98.80.-k,04.50.+h,11.10.Kk,11.10.Wx

\footnotetext{
${ }^{1}$ Electronic mail: nojiri@nda.ac.jp, snojiri@yukawa.kyoto-u.ac.jp

${ }^{2}$ Electronic mail: odintsov@ieec.fcr.es Also at TSPU, Tomsk, Russia
} 
1.Introduction. The recent astrophysical data $[1,2]$ indicate to the fact that current universe mainly consists of dark energy. Theoretically, it is absolutely dark what this dark energy should be, as any of proposed candidates is not free of the internal problems. Instead of the introduction of some mysterious fluid with large negative pressure or the fine-tuning the cosmological constant or whatever else ... one may think on the gravitational alternative for the dark energy. This leads to the modification of the gravity at current universe with the simplest possibility: $1 / R$ term $[3,4,5]$. It is natural to expect that such (or similar) effective gravity may be produced by string/M-theory/braneworld [6].

The study of the (effective) modified gravity with the terms which grow when curvature decreases shows that such theory may produce the current cosmic acceleration[3]. Unfortunately, it contains the number of instabilities $[7,8,12]$. It has been shown [14] that further modification of the modified gravity by $R^{2}$ or other higher derivative terms may help to resolve the above instabilities. Palatini form of $1 / R$ gravity $[9,10,11]$ does not contain such instabilities too and also produces the current cosmic acceleration. However, new instabilities caused by quantum effects may appear in Palatini form $[11,15]$. The variants of modified gravity with $R^{2}$ or $\ln R$ terms have been considered in metric form $[14,16]$ as well as in Palatini version [15]. Having in mind, that instabilities may be avoided [14, 16] it could be the right time to think on physical consequences and further development of modified gravity.

One immediate conclusion from the form of $1 / R$ modified gravity is that universe is never minkowskii one. In other words, there is minimal curvature of the universe somehow similar to maximal Hagedorn temperature of thermal string universe. The infinite time is required to reach the singularity point $R=0$ at the universe evolution. It should exist the mechanism which makes impossible the close approach to the minimal curvature ${ }^{3}$. Otherwise, the universe becomes highly instable. It could be that quantum effects are very important near the minimal curvature. In other words, one can conjecture the quantum gravity model where some kind of higher derivative gravity is ultraviolet fixed point of the renormalization group flow. The inflation oc-

\footnotetext{
${ }^{3}$ One can suggest the models where Lagrangian is growing with the decrease of the curvature but it takes finite value at $R=0$. The minimal curvature does not appear in such models.
} 
curs at this phase. The intermediate Einstein gravity is some (instable) transitory regime. Finally, some version of modified gravity with $1 / R^{n}$ is infrared fixed point where cosmic acceleration occurs. RG flow should define the universe evolution and control the coefficients of the corresponding effective gravity (fixed points), as well as the value for the minimal curvature. Of course, it is not so easy to develop the quantative picture for above construction. Nevertheless, one can study some elements towards to this direction.

2.Modified gravity with minimal curvature and cosmic acceleration. Let us start from the quick review of modified gravity formulation. The general 4-dimensional action is:

$$
S=\frac{1}{\kappa^{2}} \int d^{4} x \sqrt{-g} F(R)
$$

Here $R$ is the scalar curvature and $F(R)$ is some arbitrary function. Introducing the auxiliary fields $A$ and $B$, one may rewrite the action (1) as following:

$$
S=\frac{1}{\kappa^{2}} \int d^{4} x \sqrt{-g}\{B(R-A)+F(A)\} .
$$

One may eliminate $B$ and obtain

$$
S=\frac{1}{\kappa^{2}} \int d^{4} x \sqrt{-g}\left\{f^{\prime}(A)(R-A)+f(A)\right\} .
$$

By using the conformal transformation

$$
g_{\mu \nu} \rightarrow \mathrm{e}^{\sigma} g_{\mu \nu}
$$

with

$$
\sigma=-\ln F^{\prime}(A)
$$

the action (3) is rewritten as the Einstein frame action (see also [17])

$$
S_{E}=\frac{1}{\kappa^{2}} \int d^{4} x \sqrt{-g}\left(R-\frac{3}{2} g^{\rho \sigma} \partial_{\rho} \sigma \partial_{\sigma} \sigma-V(\sigma)\right) .
$$

Here

$$
V(\sigma)=\mathrm{e}^{\sigma} G\left(\mathrm{e}^{-\sigma}\right)-\mathrm{e}^{2 \sigma} F\left(G\left(\mathrm{e}^{-\sigma}\right)\right)=\frac{A}{F^{\prime}(A)}-\frac{F(A)}{F^{\prime}(A)^{2}}
$$


This is the standard form of the scalar-tensor theory where scalar field is fictitious one.

Now one can put some restrictions to the late time form of the effective potential. For instance, let us assume that when $A$ is small $F(A)$ behaves as

$$
F(A) \sim \gamma A^{-n}\left(\ln \frac{A}{\mu^{2}}\right)^{m} .
$$

Here we restrict $n$ by $n>-1$ ( $m$ is arbitrary).

Let us assume the FRW metric in the physical (Jordan) frame is:

$$
d s^{2}=-d t^{2}+\hat{a}(t)^{2} \sum_{i, j=1}^{3} \hat{g}_{i j} d x^{i} d x^{j}
$$

In the Einstein frame, the FRW equation looks like

$$
3 H_{E}^{2}=\frac{3}{4} \dot{\sigma}^{2}+\frac{1}{2} V(\sigma)
$$

Here we distinguish the quantities in the Einstein frame by the subscript $E$. The Hubble parameter $H_{E}$ is now defined by $H_{E} \equiv \frac{\dot{\hat{a}}_{E}}{\hat{a}_{E}}$. On the other hand, the equation derived by the variation over $\sigma$ is

$$
0=3\left(\ddot{\sigma}+3 H_{E} \dot{\sigma}\right)+V^{\prime}(\sigma)
$$

Then when $A$ is small, one obtains [16]

$$
t \sim t_{E}^{\frac{1}{n+2}}, \quad a_{E} \sim t_{E}^{\frac{3(n+1)^{2}}{(n+2)^{2}}}, a \sim t^{\frac{(n+1)(2 n+1)}{n+2}} .
$$

This does not depend on $m$. It takes infinite time in order that the universe reaches $R=0$. Therefore if we start from positive curvature $R>0$, the universe does not go to the region of negative curvature $R<0$.

Since $H^{2}=\frac{\kappa^{2}}{6} \rho$, the energy density $\rho$ corresponding to $\sigma$ may be defined as $\rho=\frac{\rho_{0}}{t^{2}}$. Here $\rho_{0}$ is a constant. Denoting the pressure of $\sigma$ by $p$ and substituting the above expressions of $H$ and $\rho$ to the conservation law for the energy-momentum tensor

$$
0=\dot{\rho}+3 H(\rho+p)
$$


we find the effective $w=\frac{p}{\rho}$ for $\sigma$-field

$$
w=-\frac{6 n^{2}+7 n-1}{3(n+1)(2 n+1)} .
$$

From (12), the condition that the universe could accelerate is $\frac{(n+1)(2 n+1)}{n+2}>$ 1. Clearly, the effective dark energy $w$ may be within the existing bounds.

It has been mentioned in ref.[12] that $1 / R$ model which is equivalent to some scalar-tensor gravity is ruled out as realistic theory due to the constraints to such theories. As the coupling of $\sigma$ with matter is not small [11], we now investigate the square of scalar mass, which is proportional to $V^{\prime \prime}(\sigma)$.

One may consider the case that the present universe corresponds to the minimum $A=A_{0}$ of $V(A)$, where

$$
V^{\prime}\left(A_{0}\right)=0, \quad V^{\prime \prime}\left(A_{0}\right)>0 .
$$

Then

$$
\left.\frac{d^{2} V(\sigma)}{d \sigma^{2}}\right|_{A=A_{0}}=\left.\left\{\left(\frac{d \sigma}{d A}\right)^{-2} \frac{d^{2} V(A)}{d A^{2}}\right\}\right|_{A=A_{0}} .
$$

If we adjust the parameters in $F(R)$ so that

$$
\left.\frac{d \sigma}{d A}\right|_{A=A_{0}}=0
$$

the effective mass of $\sigma$ becomes big and the scalar field $\sigma$ decouples. Then the obtained theory does not conflict with the cosmological observations, say, the solar system observations[13].

Let us now address the question of minimal curvature of the universe. To be specific, we start from the variant of the model [3]:

$$
S=\frac{1}{\kappa^{2}} \int d^{4} x \sqrt{-g}\left(R-\frac{a}{R-R_{0}}\right) .
$$

Here $R_{0}$ is a small constant (minimal curvature). If we assume that the Ricci tensor $R_{\mu \nu}$ is covariantly constant, the equation of motion corresponding to the action (18) is given by

$$
0=R-\frac{2 a}{R-R_{0}}-\frac{a R}{\left(R-R_{0}\right)^{2}} .
$$


The solution of (20) is given by

$$
\begin{aligned}
R & =\frac{2}{3} R_{0}+\alpha_{+}+\alpha_{-}, \frac{2}{3} R_{0}+\alpha_{+} \zeta+\alpha_{-} \zeta^{2}, \frac{2}{3} R_{0}+\alpha_{+} \zeta^{2}+\alpha_{-} \zeta \\
\zeta & \equiv \mathrm{e}^{i \frac{2 \pi}{3}}, \\
\alpha_{ \pm}^{3} & =-\frac{R_{0}^{3}}{27} \pm i \sqrt{a\left(a^{2}+\frac{R_{0}^{2}}{3} a+\frac{R_{0}^{4}}{27}\right)} .
\end{aligned}
$$

In case that $R_{0}^{2} \ll a$, the three solutions in (20) behave as

$$
R \sim \pm \sqrt{a}+\frac{2}{3} R_{0}+\mathcal{O}\left(R_{0}^{2}\right), \quad \frac{2}{3} R_{0}+\mathcal{O}\left(R_{0}^{2}\right)
$$

which reproduces the results in [3]. On the other hand, if $a \ll R_{0}^{2}$

$$
R \sim R_{0}
$$

After introducing an auxilliary field $A$

$$
S=\frac{1}{\kappa^{2}} \int d^{4} x \sqrt{-g}\left(\left(1+\frac{a}{\left(A-R_{0}\right)^{2}}\right)(R-A)+A-\frac{a}{A-R_{0}}\right),
$$

the action (18) can be further rewritten in a scalar-tensor form [12] with the potential:

$$
V(\sigma)=\frac{a\left(2 A-R_{0}\right)\left(A-R_{0}\right)^{2}}{\left\{\left(A-R_{0}\right)^{2}+a\right\}^{2}} .
$$

Here a metric tensor $g_{\mu \nu}$ is rescaled by

$$
g_{\mu \nu} \rightarrow \mathrm{e}^{\sigma} g_{\mu \nu}
$$

Using equations of motion, one can identify $A$ with the scalar curvature $R$ in (18). The potential $V(\sigma)(24)$ has two maxima when

$$
A=\frac{2}{3} R_{0}+\alpha_{+}+\alpha_{-}, \quad \frac{2}{3} R_{0}+\alpha_{+} \zeta^{2}+\alpha_{-} \zeta,
$$

and a minimum

$$
A=\frac{2}{3} R_{0}+\alpha_{+} \zeta+\alpha_{-} \zeta^{2}
$$


which correspond to the solutions in $(20) . V(\sigma)$ has one more extra minimum when

$$
A=R_{0}
$$

which appears since the mapping from $A$ to $\sigma$ becomes singular when $A=R_{0}$. For large $A, V(\sigma)$ behaves as

$$
V(\sigma) \sim \frac{2 a}{A}
$$

The potential $V(\sigma)$ vanishes at $A=\frac{R_{0}}{2}$ and $A=R_{0}$. The latter corresponds to the minimum in (28). However, it is not difficult to show that the infinite time is necessary to reach the state with $A=R_{0}$. Hence, the curvature approaches to the minimal one but never reaches it. On the same time, the cosmic acceleration naturally occurs in such a model.

3. Conformal anomaly resolution of the instabilities. Let us consider the role of quantum effects to the FRW universe evolution and instabilities of above modified gravity. For simplicity we take into account the quantum effects of conformally invariant fields. That may be done by adding the trace anomaly induced action $W$ to the classical action $S$ (18):

$$
\begin{aligned}
W= & b \int d^{4} x \sqrt{-\widetilde{g}} \widetilde{F} \widetilde{A} \\
& +b^{\prime} \int d^{4} x \sqrt{-\widetilde{g}}\left\{\widetilde{A}\left[2 \widetilde{\square}^{2}+\widetilde{R}_{\mu \nu} \widetilde{\nabla}_{\mu} \widetilde{\nabla}_{\nu}-\frac{4}{3} \widetilde{R}^{2}+\frac{2}{3}\left(\widetilde{\nabla}^{\mu} \widetilde{R}\right) \widetilde{\nabla} \mu\right] \widetilde{A}\right. \\
& \left.+\left(\widetilde{G}-\frac{2}{3} \widetilde{\square} \widetilde{R}\right) \widetilde{A}\right\} \\
& -\frac{1}{12}\left\{b^{\prime \prime}+\frac{2}{3}\left(b+b^{\prime}\right)\right\} \int d^{4} x \sqrt{-\widetilde{g}}\left[\widetilde{R}-6 \widetilde{\square} \widetilde{A}-6\left(\widetilde{\nabla}{ }_{\mu} \widetilde{A}\right)\left(\widetilde{\nabla}^{\mu} \widetilde{A}\right)\right]^{2}(30)
\end{aligned}
$$

In (30), one chooses the metric as

$$
g_{\mu \nu}=\mathrm{e}^{2 \widetilde{A}} \widetilde{g}_{\mu \nu}
$$

and we specify the quantities with $\widetilde{g}_{\mu \nu}$ by using $\sim$. $G(\widetilde{G})$ and $F(\widetilde{F})$ are the Gauss-Bonnet invariant and the square of the Weyl tensor. ${ }^{4}$

${ }^{4}$ We use the following curvature conventions:

$$
R=g^{\mu \nu} R_{\mu \nu}
$$


In the effective action (30), with $N$ scalar, $N_{1 / 2}$ spinor, $N_{1}$ vector fields, $N_{2}$ (=0 or 1$)$ gravitons and $N_{\mathrm{HD}}$ higher derivative conformal scalars, $b$ and $b^{\prime}$ are given by

$$
\begin{aligned}
& b=\frac{N+6 N_{1 / 2}+12 N_{1}+611 N_{2}-8 N_{\mathrm{HD}}}{120(4 \pi)^{2}} \\
& b^{\prime}=-\frac{N+11 N_{1 / 2}+62 N_{1}+1411 N_{2}-28 N_{\mathrm{HD}}}{360(4 \pi)^{2}} .
\end{aligned}
$$

In principle, $b^{\prime \prime}$ may be changed by the finite renormalization of local counterterm in gravitational effective action. Note that such anomaly induced effective action is the main component of trace anomaly driven inflation [18]. One may choose the metric $\widetilde{g}_{\mu \nu}$ to be a flat one:

$$
\widetilde{g}_{\mu \nu} d x^{\mu} d x^{\nu}=-d \tau^{2}+\sum_{i=1,2,3}\left(d x^{i}\right)^{2} .
$$

The total action $S+W$ has the following form

$$
\begin{aligned}
S+W= & \frac{1}{\kappa^{2}} \int d^{4} x \mathrm{e}^{2 \widetilde{\sigma}}\left(R-\frac{a}{R-R_{0}}\right) \\
& +\int d^{4} x\left[\frac{b^{\prime}}{2} \widetilde{\sigma} \widetilde{\square}^{2} \widetilde{\sigma}-\frac{1}{12}\left\{b^{\prime \prime}+\frac{2}{3}\left(b+b^{\prime}\right)\right\} \mathrm{e}^{2 \widetilde{\sigma}} R^{2}\right] .
\end{aligned}
$$

Here $\widetilde{\sigma}=2 \widetilde{A}$ and the scalar curvature $R$ is given by

$$
R=\mathrm{e}^{-\widetilde{\sigma}}\left(-3 \widetilde{\square} \widetilde{\sigma}-\frac{3}{2} \widetilde{g}^{\mu \nu} \partial_{\mu} \widetilde{\sigma} \partial_{\nu} \widetilde{\sigma}\right) .
$$

The equation of motion is

$$
\begin{gathered}
0=\frac{1}{\kappa^{2}}\left[\mathrm{e}^{2 \widetilde{\sigma}}\left(R-\frac{3 a}{R-R_{0}}-\frac{a R_{0}}{\left(R-R_{0}\right)^{2}}\right)+3 a \widetilde{g}^{\mu \nu} \partial_{\mu}\left(\mathrm{e}^{\widetilde{\sigma}} \partial_{\nu}\left(\frac{1}{\left(R-R_{0}\right)^{2}}\right)\right)\right] \\
+b^{\prime} \widetilde{\square}^{2} \widetilde{\sigma}+\frac{1}{2}\left\{b^{\prime \prime}+\frac{2}{3}\left(b+b^{\prime}\right)\right\} \widetilde{g}^{\mu \nu} \partial_{\mu}\left(\mathrm{e}^{\widetilde{\sigma}} \partial_{\nu} R\right) . \\
R_{\mu \nu}=R_{\mu \lambda \nu}^{\lambda} \\
R_{\mu \rho \nu}^{\lambda}=-\Gamma_{\mu \rho, \nu}^{\lambda}+\Gamma_{\mu \nu, \rho}^{\lambda}-\Gamma_{\mu \rho}^{\eta} \Gamma_{\nu \eta}^{\lambda}+\Gamma_{\mu \nu}^{\eta} \Gamma_{\rho \eta}^{\lambda} \\
\Gamma_{\mu \lambda}^{\eta}=\frac{1}{2} g^{\eta \nu}\left(g_{\mu \nu, \lambda}+g_{\lambda \nu, \mu}-g_{\mu \lambda, \nu}\right) .
\end{gathered}
$$


One assumes the spacetime is deSitter space

$$
\begin{aligned}
d s^{2} & =\frac{l^{2}}{\tau^{2}}\left(-d \tau^{2}+\sum_{i=1,2,3}\left(d x^{i}\right)^{2}\right) \\
& =-d t^{2}+\mathrm{e}^{\frac{2 t}{l}} \sum_{i=1,2,3}\left(d x^{i}\right)^{2} \\
\tau & =-l \mathrm{e}^{-\frac{t}{l}}, \\
R & =\frac{12}{l^{2}} .
\end{aligned}
$$

Then the equation (36) has the following form:

$$
0=\frac{l^{4}}{\kappa^{2}}\left\{\frac{12}{l^{2}}-\frac{3 a}{\frac{12}{l^{2}}-R_{0}}-\frac{a R_{0}}{\left(\frac{12}{l^{2}}-R_{0}\right)^{2}}\right\}+12 b^{\prime} .
$$

Taking

$$
R=R_{1} \equiv \frac{12}{l^{2}},
$$

when $b^{\prime}=$ 0, Eq.(38) reproduces Eq.(19). On the other hand, when $a=0$, Eq.(38) reproduces the anomaly driven inflation [18].

The perturbation from the solution of (38) is:

$$
\widetilde{\sigma}=\ln \frac{l^{2}}{\tau^{2}}+\delta \sigma
$$

Here $l^{2}$ satisfies eq.(38). Then by linearizing Eq.(36) and by using Eq.(38), we obtain

$$
\begin{aligned}
& 0=\frac{1}{\kappa^{2}}\left[-\frac{24 \kappa^{2} b^{\prime}}{\tau^{4}} \delta \sigma+\frac{l^{4}}{\tau^{4}}\left(1+\frac{3 a}{\left(R_{1}-R_{0}\right)^{2}}+\frac{2 a R_{0}}{\left(R_{1}-R_{0}\right)^{3}}\right) \delta R\right] \\
& +b^{\prime} \tilde{\square} \delta \sigma+\left[-\frac{6 a}{\kappa^{2}\left(R_{1}-R_{0}\right)^{3}}+\frac{1}{2}\left\{b^{\prime \prime}+\frac{2}{3}\left(b+b^{\prime}\right)\right\}\right] \widetilde{g}^{\mu \nu} \partial_{\mu}\left(\frac{l^{2}}{\tau^{2}} \partial_{\nu} \delta R\right) .
\end{aligned}
$$

Here

$$
\delta R=-\frac{12}{l^{2}} \delta \sigma+\frac{\tau^{2}}{l^{2}}\left(-3 \widetilde{\square} \delta \sigma+\frac{6}{\tau} \partial_{\tau} \delta \sigma\right) .
$$


By using the ambiguity of the renormalization for $b^{\prime \prime}$, we may choose (as kind of renormalization condition)

$$
0=-\frac{6 a}{\kappa^{2}\left(R_{1}-R_{0}\right)^{3}}+\frac{1}{2}\left\{b^{\prime \prime}+\frac{2}{3}\left(b+b^{\prime}\right)\right\} .
$$

When $\tau \rightarrow$ 0, Eq.(42) behaves as

$$
\delta R \sim-\frac{12}{l^{2}} \delta \sigma
$$

Then the terms inside the first bracket [ ] (41) dominate when $\tau \rightarrow 0$ and

$$
\delta \sigma \sim 0
$$

The limit $\tau \rightarrow 0$ corresponds to $t \rightarrow+\infty$ in (37), which is the late time (or present time) of the universe. Eq.(45) tells that the perturbation is strongly suppressed. Then the instabilities as in $[8,11,12]$ do not occur. In fact, the quantum effects produce the higher derivative terms which may suppress the instabilities in the same way as in ref.[14].

We should stress that, if there is no the quantum correction, the theory becomes inconsistent with the present cosmology due to the instabilities. As $\kappa^{-2} \sim\left(10^{19} \mathrm{GeV}\right)^{2}=\left(10^{28} \mathrm{eV}\right)^{2}$, if we assume $R_{1} \sim R_{0} \sim \sqrt{a} \sim\left(10^{-33} \mathrm{eV}\right)^{2}$, which corresponds to the present acceleration of the universe, we find

$$
b^{\prime \prime}+\frac{2}{3}\left(b+b^{\prime}\right) \sim\left(10^{28+33}\right)^{2}=10^{122},
$$

which does not seem to be realistic. One may consider, however, the case that $a \ll R_{0}^{2}$ as in (22). If $a \sim 10^{-155} \mathrm{eV}^{2}$, the parameters $b, b^{\prime}$ and $b^{\prime \prime}$ can be of the order of unity.

In [12], it has been shown that the general modified gravity can be rewritten in the form of the scalar-tensor theory. As the order of the coupling of the extra scalar field with matter field is always the same [11], there seems to be a conflict with the solar system observations[13] if the scalar field is massless or very light. If the scalar field is heavy, however, the scalar field decouples and there is no problem. The fluctuation of the scalar field corresponds to $\delta \sigma$ in (40). Then since the scalar field $\delta \sigma$ decouples if the condition (43) is satisfied, which tells that the effective mass of $\delta \sigma$ becomes very heavy, the 
quantum effects help to avoid the problem with the solar system tests. Thus, the role of quantum effects is in resolution of the instabilities and in making for theory even more difficult the approach to minimal curvature phase which is highly instable.

Indeed, what happens when $R \sim R_{0}$ where the action (18) becomes sigular at $R=R_{0}$ (minimal curvature when $R$ approaches to it from above). Then in (36), the terms including the inverse power of $\delta R=R-R_{0}$ dominate and we obtain:

$$
0 \sim-\frac{a R_{0} \mathrm{e}^{2 \sigma}}{\left(R-R_{0}\right)^{2}}-3 a \partial_{\tau}\left(\frac{l_{0}^{2}}{\tau^{2}} \partial_{\tau}\left(\frac{1}{\left(R-R_{0}\right)^{2}}\right)\right) .
$$

Here the length parameter $l_{0}$ is given by

$$
R_{0}=\frac{12}{l_{0}^{2}}
$$

Assuming

$$
\delta R \sim \alpha \tau^{\beta}
$$

with constants $\alpha$ and $\beta$, one finds

$$
\beta=-\frac{1}{2}, \quad-2
$$

$\sigma$ may be written as

$$
\tilde{\sigma}=\ln \frac{l_{0}^{2}}{\tau^{2}}+\delta \sigma(\tau) .
$$

The first term in (51) gives the constant curvature of deSitter spacetime $R=R_{0}$. We now assume the second term in (51) is rather small compared with the first term. Then $\delta R$ is given in a form similar to (42):

$$
\delta R=-\frac{12}{l_{0}^{2}} \delta \sigma+\frac{\tau^{2}}{l_{0}^{2}}\left(3 \partial_{\tau}^{2} \delta \sigma+\frac{6}{\tau} \partial_{\tau} \delta \sigma\right) .
$$

From (49) and (50) it follows

$$
\begin{aligned}
\delta \sigma \sim \alpha^{\prime} \tau^{\beta}, \quad \beta & =-\frac{1}{2}, \quad-2 \\
\alpha^{\prime} & =\frac{l_{0}^{2} \alpha}{3\left(\beta^{2}+\beta-4\right)} .
\end{aligned}
$$


Since $\delta \tau$ and $\delta R$ become large when $\tau \rightarrow 0$, the above approximation breaks down for large $t$. As the cosmological time $t$ is given by (37), $R \sim R_{0}$ is approximately solution when $t \rightarrow-\infty$ but the perturbation grows up with $t$. If one changes the direction of the $t$, we may obtain a solution where the curvature $R$ approaches to the critical value $R_{0}$, although it takes infinite time in order for $R$ to reach $R_{0}$. In such a model, as the spacetime is asymptotically deSitter, we have effective cosmological constant and we find that $w \sim 1$. Here $w$ is the ratio of the effective pressure $p$ and the effective energy density $\rho: w=\frac{p}{\rho} . \delta \sigma$ in (53) will give a correction to $w$. Including the contribution from $\delta \sigma$, since $d t=\mathrm{e}^{\frac{\sigma}{2}} d \tau$, the cosmological time $t$ is modified as

$$
t \sim-l\left(\ln \tau+\frac{\alpha^{\prime}}{2 \beta} \tau^{\beta}\right)
$$

or

$$
\tau \sim \mathrm{e}^{-\frac{t}{l}}\left(1-\frac{\alpha^{\prime}(1+\beta)}{2 \beta} \mathrm{e}^{-\frac{\beta t}{l}}\right) .
$$

For FRW metric: $d s^{2}=-d t^{2}+a^{2}(t) \sum_{i=1,2,3}\left(d x^{i}\right)^{2}$, we find

$$
a \sim l \mathrm{e}^{\frac{t}{l}}\left(1+\frac{\alpha^{\prime}(1+\beta)}{2 \beta} \mathrm{e}^{-\frac{\beta t}{l}}\right)
$$

Since the Hubble constant is given by $H=\frac{\dot{a}}{a}$ and from the FRW equations $H=\frac{\kappa^{2}}{6} \rho$ and $\dot{H}=-\frac{\kappa^{2}}{4}(\rho+p)$, we find

$$
\rho \sim \frac{6}{\kappa^{2} l^{2}}\left(1-\alpha^{\prime} l(1+\beta) \mathrm{e}^{-\frac{\beta}{l} t}\right), \quad p \sim-\rho-\frac{6}{\kappa^{2} l^{2}} \frac{\alpha^{\prime} l \beta(1+\beta)}{3} \mathrm{e}^{-\frac{\beta}{l} t} .
$$

we find

$$
w=\frac{p}{\rho}=-\left(1+\frac{\alpha^{\prime} l \beta(1+\beta)}{3} \mathrm{e}^{-\frac{\beta}{l} t}\right) .
$$

Since $\beta$ is negative, the correction from $w=-1$ is growing with the time.

4.Discussion. In summary, it is shown that recently suggested models of modified gravity contain the minimal curvature. In other words, the universe is never flat one. The infinite time is necessary in order to reach the minimal curvature in the current epoch of cosmic acceleration. It is remarkable that quantum effects of conformal fields may help in stabilizing of such gravity. 
Many more variants of modified gravity may be suggested. For instance, one may combine the modification of the gravitational action at the present epoch with the presence of the cosmological constant (also kind of dark energy). The simplest example of such theory which admits current cosmic speed-up and has no the minimal curvature is given below:

$$
F(A)=\frac{\mathrm{e}^{\beta A}-\alpha}{\beta}
$$

Here we assume $\beta>0$. Then

$$
\sigma=-\beta A=-\beta R
$$

and

$$
\begin{aligned}
V(\sigma) & =\left(A-\frac{1}{\beta}\right) \mathrm{e}^{-\beta A}+\frac{\alpha}{\beta} \mathrm{e}^{-2 \beta A} \\
& =-\frac{(\sigma+1) \mathrm{e}^{\sigma}}{\beta}+\frac{\alpha}{\beta} \mathrm{e}^{2 \sigma} .
\end{aligned}
$$

If

$$
\alpha>\frac{\mathrm{e}}{2},
$$

the potential $V$ is monotonically decreasing (increasing) function of $A(\sigma)$. If the condition (62) is satisfied, there is no deSitter space solution. The FRW equation (10) and the $\sigma$-equation (11) can be solved as a power series:

$$
\begin{aligned}
& \sigma=\sigma_{1} t_{E}+\left(\mp \frac{3 \sigma_{1}}{2} \sqrt{\frac{\sigma_{1}^{2}}{4}+\frac{\alpha-1}{6 \beta}}-\frac{\alpha-1}{3 \beta}\right) t_{E}^{2}+\mathcal{O}\left(t_{E}^{3}\right) \\
& H_{E}= \pm \sqrt{\frac{\sigma_{1}^{2}}{4}+\frac{\alpha-1}{6 \beta}}+\left(-\frac{3}{4} \sigma_{1} \pm \frac{(\alpha-1)\left(\sigma_{1}-1\right)}{6 \beta \sqrt{\frac{\sigma_{1}^{2}}{4}+\frac{\alpha-1}{6 \beta}}}\right) t_{E}+\mathcal{O}\left(t_{E}^{2}\right)
\end{aligned}
$$

Here $\sigma_{1}$ is a constant of integration (one more constant of the integration can be absorbed into the constant shift of $\left.t_{E}\right)$. We may assume $\sigma_{1}<0$ so that $R>0$ in (60). As a result

$$
t \sim \mathrm{e}^{\frac{1}{2} \sigma_{1} t_{E}}, \quad a \sim \mathrm{e}^{\left(\sigma_{1}+\mp \sqrt{\frac{\sigma_{1}^{2}}{4}+\frac{\alpha-1}{6 \beta}}\right) t_{E}} \sim t^{1 \mp \sqrt{1+\frac{2(\alpha-1)}{3 \beta \sigma_{1}^{2}}}} .
$$


Then if $\alpha>1$ (or more strongly Eq.(62) is satisfied), the universe is accelerating for the branch with +-sign in (64). Note that the rate of the acceleration is dynamically determined by the constant of integration $\sigma_{1}$. The effective $w$ is found as

$$
w=-1+\frac{2}{3(1+x)}, \quad x \equiv \sqrt{1+\frac{2(\alpha-1)}{3 \beta \sigma_{1}^{2}}} .
$$

$w$ is a monotonically decreasing function of $x$. When $x \rightarrow 1$, which is the limit of $\beta \rightarrow \infty$ or $\sigma_{1} \rightarrow \infty, w \rightarrow-\frac{1}{3}$. On the other hand, when $x \rightarrow+\infty$, which is the limit of $\beta \rightarrow 0$ or $\sigma_{1} \rightarrow 0$, we obtain $w \rightarrow-1$. The equation of state satisfies

$$
-1<w<-\frac{1}{3}
$$

which may be consistent with the present observational constraint $-1.45<$ $w<-0.74$. The above example indicates that true dark energy may be the combination of the (weak) gravitational alternative for dark energy, cosmological constant and quantum models of the sort discussed in [19, 20].

Acknowledgments The research is supported in part by the Ministry of Education, Science, Sports and Culture of Japan under the grant n.13135208 (S.N.), DGI/SGPI (Spain) project BFM2000-0810 (S.D.O.), RFBR grant 0301-00105 (S.D.O.) and LRSS grant 1252.2003 .2 (S.D.O.).

\section{References}

[1] A.G. Reiss et al, Astron. J. 116 (1998) 1009; S. Perlmutter et al, Astrophys. J. 517 (1999) 565; J.L. Tonry et al, astro-ph/0305008.

[2] C.I. Bennet et al, astro-ph/0302207; C.B. Netterfield et al, Astrophys. J. 571 (2002) 604; N.W. Halverson et al, Astrophys. J. 568 (2002) 38.

[3] S.M. Carroll, V. Duvvuri, M. Trodden and M.S. Turner, astro$\mathrm{ph} / 0306438$.

[4] S. Capozzielo, S. Carloni and A. Troisi, astro-ph/0303041;

[5] S. Capozzielo, V.F. Cardone, S. Carloni and A. Troisi, astro-ph/0307018.

[6] S. Nojiri and S.D. Odintsov, hep-th/0307071, PLB, to appear. 
[7] A.D. Dolgov and M. Kawasaki, astro-ph/0307285;

[8] M.E. Soussa and R.P. Woodard, astro-ph/0308114.

[9] D.N. Vollick, astro-ph/0306630; Y. Ezawa, H. Iwasaki, Y. Ohkura, N. Yamada and T. Yano, gr-qc/0309010.

[10] X. Meng and P. Wang, astro-ph/0307354; astro-ph/0308031.

[11] E.E. Flanagan, astro-ph/0308111; gr-qc/0309015.

[12] T. Chiba, astro-ph/0307338.

[13] C.M. Will, Living Rev.Rel. 4 (2001) 4, gr-qc/0103036.

[14] S. Nojiri and S.D. Odintsov, Phys.Rev.D, to appear, hep-th/0307288.

[15] X. Meng and P. Wang, astro-ph/0308284; hep-th/0309062.

[16] S. Nojiri and S.D. Odintsov, hep-th/0308176.

[17] G. Magnano and L.M. Sokolowski, Phys.Rev. D50 (1994) 5039.

[18] A. Starobinsky, Phys.Lett. B91 (1980) 99.

[19] L. Parker, A. Raval, Phys.Rev. D60 (1999) 063512, Erratum-ibid.D67 (2003) 029901, gr-qc/9905031.

[20] E. Elizalde, J.E. Lidsey, S. Nojiri, S.D. Odintsov, to appear in Phys.Lett. B, hep-th/0307177. 